\title{
PLK2 Gene
}

National Cancer Institute

\section{Source}

National Cancer Institute. PLK2 Gene. NCI Thesaurus. Code C24818.

This gene is involved in normal cell division. 